\title{
Statistical Tests of Load-Unload Response Ratio Signals by Lattice Solid Model: Implication to Tidal Triggering and Earthquake Prediction
}

\author{
Yucang Wang ${ }^{1,2}$, Peter Mora $^{1}$, CAN Yin $^{1}$, and David Place ${ }^{1}$
}

\begin{abstract}
Statistical tests of Load-Unload Response Ratio (LURR) signals are carried in order to verify statistical robustness of the previous studies using the Lattice Solid Model (MorA et al., 2002b). In each case 24 groups of samples with the same macroscopic parameters (tidal perturbation amplitude $A$, period $T$ and tectonic loading rate $k$ ) but different particle arrangements are employed. Results of uni-axial compression experiments show that before the normalized time of catastrophic failure, the ensemble average LURR value rises significantly, in agreement with the observations of high LURR prior to the large earthquakes. In shearing tests, two parameters are found to control the correlation between earthquake occurrence and tidal stress. One is, $A /(k T)$ controlling the phase shift between the peak seismicity rate and the peak amplitude of the perturbation stress. With an increase of this parameter, the phase shift is found to decrease. Another parameter, $A T / k$, controls the height of the probability density function (Pdf) of modeled seismicity. As this parameter increases, the Pdf becomes sharper and narrower, indicating a strong triggering. Statistical studies of LURR signals in shearing tests also suggest that except in strong triggering cases, where LURR cannot be calculated due to poor data in unloading cycles, the larger events are more likely to occur in higher LURR periods than the smaller ones, supporting the LURR hypothesis.
\end{abstract}

Key words: Load-Unload Response Ratio (LURR), Lattice Solid Model (LSM), numerical simulation, tidal trigger, earthquake prediction.

\section{Introduction}

Tidal trigger of earthquakes or possible correlation between tidal forces and earthquakes aroused interest for several decades (CATTON, 1922; TsURUOKA et al., 1995; EMTER, 1997). It seems plausible that tidal forces should trigger earthquakes considering the fact that the peak rate of tidal forces may be significantly higher than average rates of tectonic stress buildup. Although there are studies reporting a positive correlation between earthquake occurrence and the earth tide (TSURUOKA

${ }^{1}$ QUAKES, Department of Earth Sciences, The University of Queensland, Qld 4072, Brisbane, Australia. E-mails: wangyc@quakes.uq.edu.au, mora@quakes.uq.edu.au, canyon@quakes.uq.edu.au, place@quakes.uq.edu.au

${ }^{2}$ LNM, Institute of Mechanics, Chinese Academy of Sciences, Beijing, 100080, China. E-mail: yin@lnm.imech.ac.cn 
et al., 1995; WiLliam and WiLCOCK, 2001; TOLSTOY et al., 2002), negative results have also been published (HEATON, 1982; RYDELEK et al., 1992; VidALE et al., 1998). This discrepancy at least suggests that no significant strong correlation has been widely observed.

The Load-Unload Response Ratio hypothesis (LURR) views this problem from a different perspective. The basic assumption of LURR is that at an earlier stage of damage a system is stable, and not sensitive to minor external disturbances, whereas when approaching the macroscopic failure, the system will have a quite different response to minor external loading and unloading. At this stage it may be possible for tidal correlation to be observed. The reason why no significant tidal triggering has been widely observed may be that the instantaneous tidal loads are not large enough to always trigger earthquakes and are not the only factor effecting earthquakes. In retrospective studies, high LURR values have been observed months to years prior to most events and intermediate-term earthquake predictions have been made (YIN et al., 2000, 2002). Recent study indicates that optimal LURR region sizes scale with magnitudes of forthcoming events, similar to Accelerating Moment Release (AMR) observations (BOWMAN et al., 1998), which suggest that both LURR and AMR may be used as a measure of the proximity to a critical state, and may have the same underlying physical mechanism (YIN et al., 2002).

As a first step towards understanding the underlying physical mechanism for the LURR observations, numerical studies are conducted using the Lattice Solid Model (LSM) (MORA et al., 2002b). The preliminary simulations reproduce signals similar to those observed in earthquake prediction practice with a high LURR value followed by a sudden drop prior to macroscopic failure of the sample, suggesting that LURR provides a good predictor for catastrophic failure in elastic-brittle systems. Since random-sized particles are involved in the model, different random configurations and parameters are needed to test statistical robust and possible dependence on the parameters.

In this paper, we first present the statistical features of LURR under uni-axial compression using the Lattice Solid Model. Since shearing simulations generate more events, in the second part, we investigate the effects of parameters on earthquake occurrence and statistical features of LURR under shearing loading.

\section{Statistical Tests of LURR under Uni-axial Compression Using Different Random Configurations}

As in the previous study (MORA et al., 2002b), the model is initialized as a heterogeneous 2-D block made up of random-sized particles with diameters ranging from 0.2 to 1 model units. The system is subjected to uni-axial compression from rigid driving plates on the upper and lower edges of the model. Experiments are conducted using both strain and stress control to load the plates. The detailed 
calibration and fracture orientation can be found in the previous papers (MORA et al., 2002a, 2002b; PlACE et al., 2002). A sinusoidal stress perturbation is added to the constant loading rate to simulate loading and unloading cycles induced by tidal forces

$$
\Delta \sigma_{z z}=A \cos (2 \pi t / T)
$$

where $T$ and $A$ are period and amplitude of tidal stress. Thus total stress is $\sigma_{z z}=$ $\Delta \sigma_{z z}+k t, k$ represents loading rate of tectonic stress $\sigma_{z z}$. LURR is calculated using

$$
L U R R=E^{+} / E^{-}
$$

where $E^{+}$and $E^{-}$respectively denote the cumulative seismic energy release during loading and unloading within a given time window. The cumulative seismic energy release is obtained by summing total kinetic energy released during all loading or unloading cycles within the specified time window, where we define loading when $d \sigma_{z z} / d t \geqslant 0$, and unloading when $d \sigma_{z z} / d t<0$. The total kinetic energy release at any given instant $t$ is the sum of the kinetic energy within the system and the energy lost to the artificial viscosity energy prior to time $t$ (MoRA et al., 2002b).

In this test, 24 groups of simulations with the same parameters of $T, A$ and $k$ but different configurations of random particles are conducted. LURR values are calculated for each sample in the same way as previous work (MORA et al., 2002b). Due to sample specificity (XIA et al., 1996; BAI et al., 2000), the fracture pattern and catastrophic failure times are different for each sample. Therefore failure times are normalized to unit for comparison and average. Figure 1 shows plots of the averaged LURR values from 24 groups versus normalized time for the same parameters used in the previous study. One can see that the averaged LURR begins to rise from $t=0.5$, and reaches its peak at $t=0.9$ and then drops just before the main event at

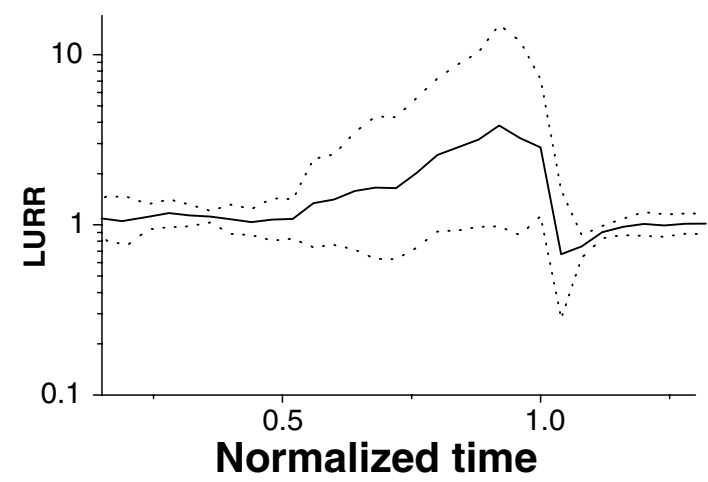

Figure 1

Evolution of ensemble average LURR computed from 24 samples. The dotted line marks the standard deviations. Parameters are: $k=30 \mathrm{MPa} / 100,000$ time steps, $T=4000$ time steps and $A=0.96 \mathrm{MPa}$. 
$t=1.0$. After $t=1.0$, although there are many events, it is flat. It is also noted that the standard deviations of LURR increase before the main event.

Due to the limitation of computer power, it is unfeasible to scan all the parameter range, simulations are conducted using values $T, A$ and $k$ with a factor of five larger and smaller. Similar results suggest that our previous results are statistically robust.

Figure 2 shows the evolution of averaged cumulative energy release before the catastrophic failure using the same 24 groups of samples as in Figure 1. The dotted line is the fitted time-to-failure function according to $\Omega(t)=A+B\left(t_{f}-t\right)^{m}$ (BUFE et al., 1993), here $t_{f}$ is set to 1.0. Apparent accelerated energy release is observed, suggesting that both high LURR and AMR appear in these tests just before the main fracture.

There are still very few events before the catastrophic failure (generally 20-30 events) under uni-axial compression. In order to generate more events before the larger earthquakes, shearing tests are used in the next section.

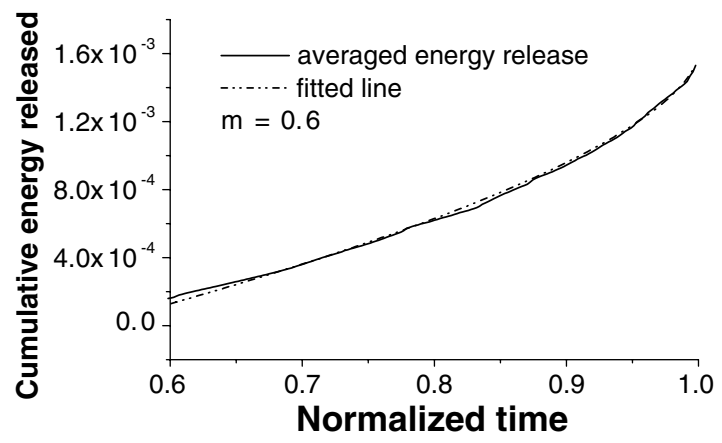

Figure 2

Evolution of ensemble average cumulative energy release. The dotted line is fitted time-to-failure function according to $\Omega(t)=A+B\left(t_{f}-t\right)^{m}$ (BuFE et al., 1993), $t_{f}$ is set to 1.0. Parameters are the same as above.

\section{Statistical Test of LURR under Shearing Tests}

\subsection{Model Description}

In this test the model consists of a central fault sandwiched between elastic regions which are attached to rigid driving plates at their outer boundaries (Fig. 3). The fault region is composed of random-sized particles. The surface roughness of the fault is controlled by two parameters: a fractal dimension $D$ and height of asperities $H(0.5$ and 1.6 in this simulation). Between the fault particles, simple frictional force with magnitude proportional to the normal force is employed (Place et al., 2001; MorA et al., 2002a). Boundary condition is circular in a horizontal direction. The shearing is achieved by moving the driving plates at a moderate and constant rate which 


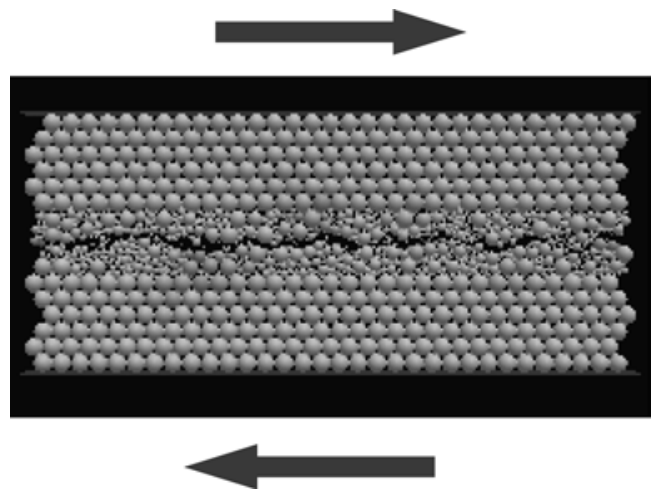

Figure 3

Snapshot of a shearing test. Boundary conditions are circular in the horizontal direction. A constant normal stress of $150 \mathrm{MPa}$ is applied at the upper and lower rigid driving plates.

models the slow tectonic loading while maintaining a constant normal stress of $150 \mathrm{MPa}$. Similarly, a slight sinusoidal perturbation is added to the slow tectonic loading, simulating tidal stress. The model allows rupture to occur along the internal surface within the fault region, modeling the slip and stick process (and even breaking of asperities). Thus this model can be considered as a simplified model for an interacting fault system containing a long single fault.

\subsection{Effects of the Parameters on Earthquake Occurrence}

To simulate the effects of tidal stress on earthquake occurrence, the parameters cannot be chosen arbitrarily, but according to the following conditions

$$
\begin{aligned}
& \left|\Delta \sigma_{z z}\right| \ll \sigma_{z z} \\
& \frac{d\left|\Delta \sigma_{z z}\right|}{d t} \gg k \\
& T_{e} \ll T \ll T_{L} .
\end{aligned}
$$

where $T_{e}$ is the earthquake rupture duration and $T_{L}$ is the average time interval between large earthquakes. Two dimensionless parameters are introduced to describe the conditions above, $k_{1}=\frac{\left|\Delta \sigma_{z z}\right|}{\sigma_{z z}}, k_{2}=\frac{d\left|\Delta \sigma_{z z}\right|}{d t} / k=\frac{2 \pi A}{T k}$ Equations (3) and (4) require $k_{2}>>1$, and $k_{1} \ll 1$, or $T k / 2 \pi \ll A \ll k t$, where $\mathrm{t}$ is the tectonic loading time.

In nature, tidal stresses can be up to $0.001-0.004 \mathrm{MPa}$, with its rate of about 0.001 $\mathrm{MPa} / \mathrm{h}$ and typical period $T$ of 12 hours. The tectonic stress should be in the order of 10-100 $\mathrm{MPa}$, with long-term rate of approximately $10^{-6}-10^{-4} \mathrm{MPa} / \mathrm{h}$. so the approximate ranges of two parameters are: $k_{1}=10^{-5}-10^{-4}$ and $k_{2}=10^{1}-10^{3}$.

Due to limited computer power, it is impossible to use the observed parameters in our numerical experiments, therefore we use a higher loading rate. In this simulation, the parameters are: $A=0.06-4.8 \mathrm{MPa}, T=2000-8000$ time steps and 
$k=10^{-4}-10^{-3} \mathrm{MPa}$ per time step. Strength of the sample is found to be about $120 \mathrm{MPa}$. Thus $k_{1}=5 \times 10^{-4}-4 \times 10^{-2}$ and $k_{2}=1-100$. Note that $k_{2}<1.0$ means a constant positive $d \sigma_{z z} / d t$, and a violation of Eq. (4), therefore LURR is not calculated in this case, nonetheless the probability density functions (see below) are still carried out to compare parameter effects.

To study the effects of parameters such as $A, T$ and $k$ on earthquake occurrence, we compared the probability density function ( $\mathrm{Pdf}$ ) of modeled events versus phase angle for different parameters. Here phase angle $\theta \in[-\pi, \pi]$, and $\theta+2 \pi n=\frac{2 \pi}{T} t, n$ is the integer number of cycles and $t$ is event time.

The typical Pdf curves are shown in Figure 4. One can generally see a peak and phase shift $\phi$ between the peak seismicity rate and the peak amplitude of the perturbation stress. Detailed studies indicate that the Pdf curve is mainly controlled by two parameters, rather than $A, T$ and $k$ individually. One is $k_{2}$, controlling phase shift $\phi$. This is clearly seen in Figure 4, where three curves have different $A, T$ and $k$, but the same $k_{2}$, the Pdf peaks are found to appear in a similar place.

When $k_{2}$ is increased, the phase shifts seemingly decrease from $2 \pi$ to 0 (Fig. 5), indicating that the peak seismicity occurrence rate shifts from the position of peak perturbation rate towards that of peak perturbation.

Another parameter, $k_{3} \propto A T / k$ controls the peak height of the Pdf curves. As this parameter increases, the Pdf becomes sharper and narrower, indicating strong correlation (Fig. 6 and Fig. 4). When $k_{3}$ is too small (e.g., 0.08), however, Pdf is almost flat, indicating no apparent tidal correlation.

Therefore we can conclude that in such an elastic-brittle system, a larger amplitude of perturbation and smaller loading rate contribute stronger correlation

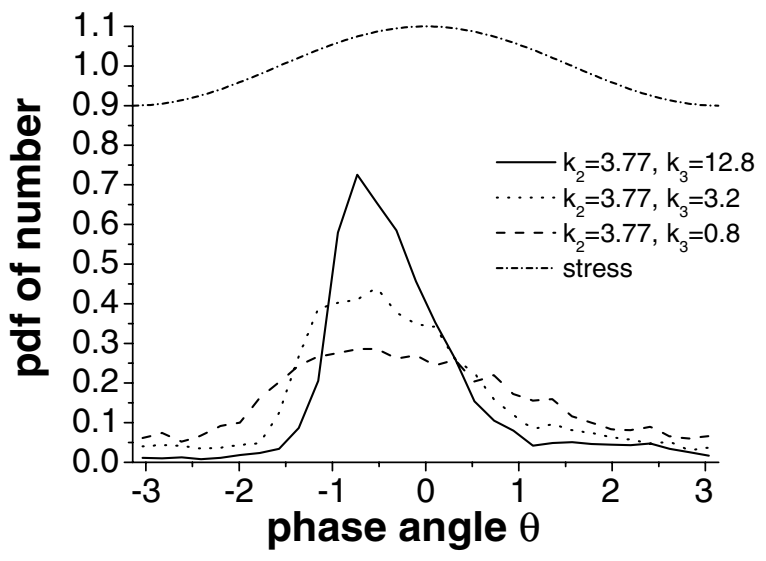

Figure 4

Plots of Pdf of events versus phase angle for different $A / k$ and $T$ although the same $k_{2}$ The perturbation stress is plotted for comparison. Parameters are: $k=5 \mathrm{Mpa} / 10000$ time step, $T=8,000$ time steps, $A=2.4$ $\operatorname{MPa}\left(k_{3}=12.8\right) ; k=5 \mathrm{MPa} / 10,000$ time step, $T=4,000$ time steps, $A=1.2 \mathrm{MPa}\left(k_{3}=3.2\right) ; k=5 \mathrm{MPa} /$ 10,000 time step, $T=2,000$ time steps, $A=0.6 \mathrm{MPa}\left(k_{3}=0.8\right)$. 


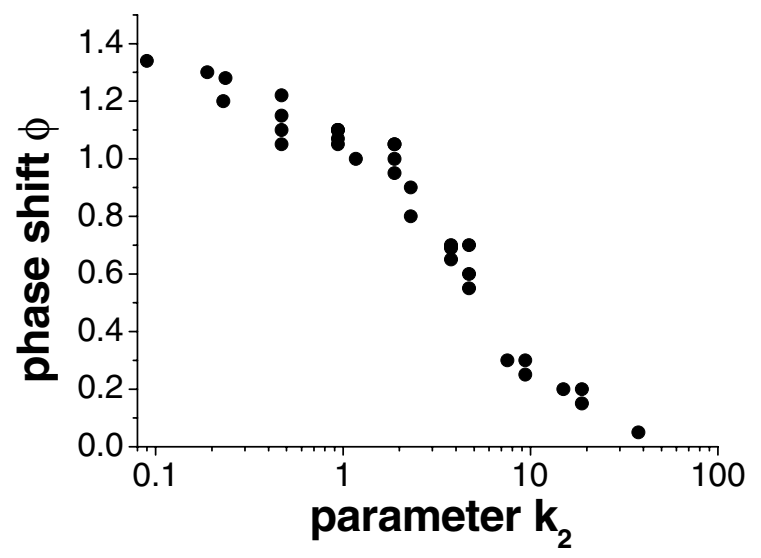

Figure 5

Dependence of phase shift $\phi$ on $k_{2}$. When $k_{2}$ increases, $\phi$ decreases, indicating a shift of the peak seismicity rate towards the position of peak tidal stress.

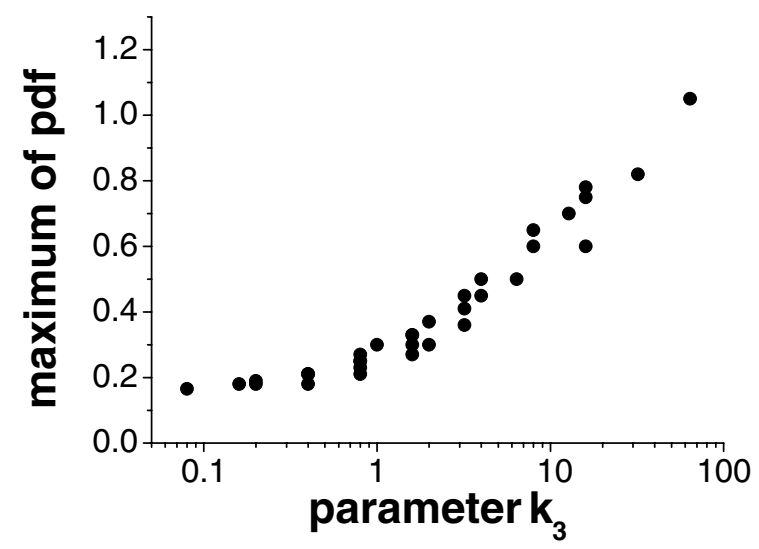

Figure 6

Relation between the peak height of the Pdf curves and parameter $k_{3}$ The larger $k_{3}$ corresponds to the higher and narrower Pdf curves, indicating stronger triggering.

between tidal stress and seismicity. Besides $A$ and $k$, the period of tidal stress $T$ also plays an important role. On one hand, decreasing $T$ makes the peak seismicity rate approach the peak of the perturbation stress. On the other hand, smaller $T$ causes the low peak height of Pdf curve, lessening the tidal effects.

\subsection{Statistics of LURR in Shearing Tests}

Since a phase shift is observed between the peak seismicity rate and the peak of the perturbation stress, loading and unloading are redefined here. In this study 
$\cos (2 \pi t / T+\phi)>0$ is defined as loading, otherwise as unloading. Meanwhile, the averaged triggering effect should be deducted in order to keep averaged LURR around 1. A parameter is defined to roughly describe the average triggering effect.

$$
\alpha=\frac{\text { total event number in loaing cycles }}{\text { total event number in unloading cycles }} \text {. }
$$

Then LURR values are calculated using

$$
L U R R=\frac{\sum E^{+} / \sum E^{-}}{\alpha}
$$

where $E^{+}$and $E^{-}$are kinetic energy released during modeled earthquake events in loading and unloading cycles respectively. Time window of $3 T$ and energy cut-off of $3 \mathrm{e}-5$ (events with energy less than $3 \mathrm{e}-5$ are employed in calculations) are used to avoid large fluctuation. Unlike the compression test, no predominant large events are found in this simulation, a different statistical method is adopted instead of normalizing the failure time into unit. For 8 groups of simulation with the same parameters, we divide the total events into two groups: the $10 \%$ largest events and the rest of the smaller ones. Then we compare Pdf versus LURR values for the two groups. Dot line in Figure 7 shows the percentage of smaller events which occur in a certain pin of LURR; we can see that Pdf is nearly random distributed. However the apparent right shift of Pdf for the 10\% largest events (solid lines) means that the larger events may occur more likely in high LURR periods and less likely in low LURR periods than the smaller ones.

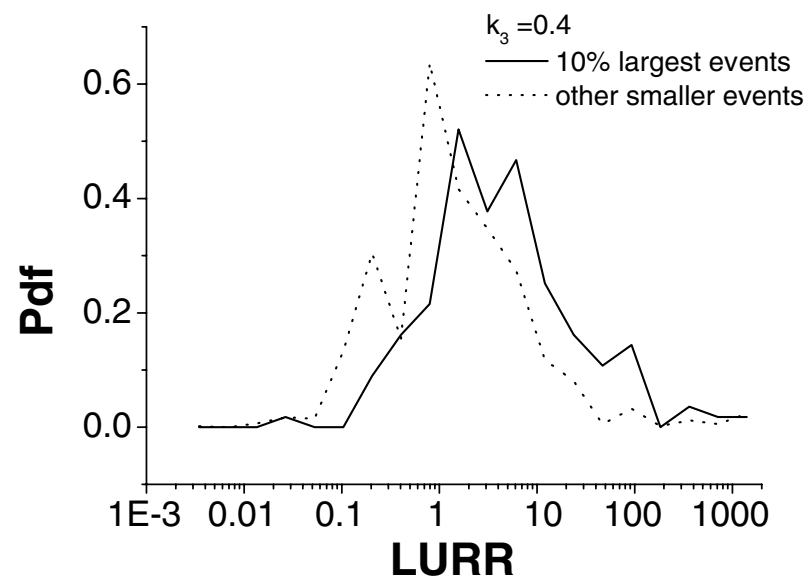

Figure 7

Pdf versus LURR for the largest $10 \%$ of events (solid) and other smaller events (dotted). $k=5 \mathrm{MPa}$ / 10,000 time step, $T=2,000$ time steps, $A=0.6 \mathrm{MPa}$ and $k_{3}=0.4, \alpha=1.31$. 


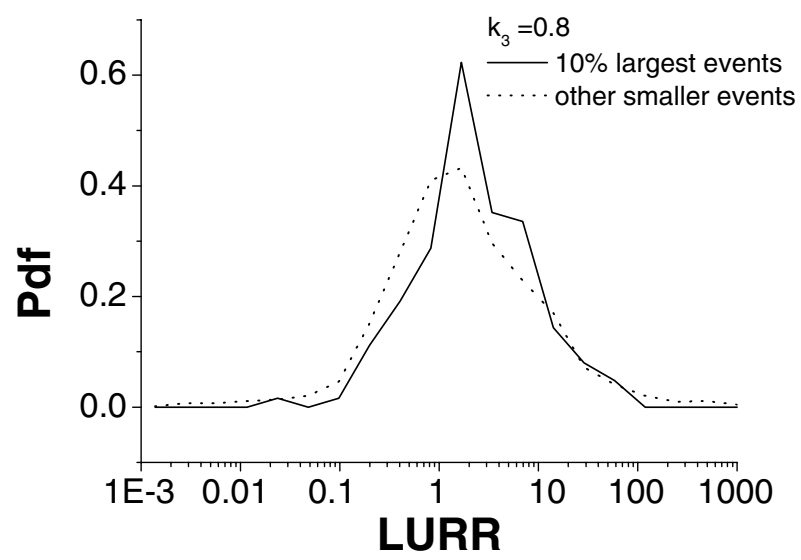

Figure 8

Pdf versus LURR for the largest $10 \%$ of events (solid) and other smaller events (dotted). $k=5 \mathrm{MPa}$ / 10,000 time step, $T=2,000$ time steps, $A=0.3 \mathrm{MPa}$ and $k_{3}=0.8, \alpha=1.45$.

Since in a strong triggering case very few events would occur in unloading cycles, making it impossible to calculate LURR. LURR is calculated only in weak triggering cases. Our statistical results show that except for strong triggering cases with large $k_{3}$ (e.g. $k_{3}>0.8$ ), similar results are observed in all other cases (Fig. 8). These results suggest that although no apparent overall tidal correlations of earthquake are widely observed, it is still possible that the large events are more likely to occur in high LURR periods.

\section{Discussions and Conclusions}

The statistical studies presented in this paper verified statistical robustness of previous results (MORE et al., 2002b). In the weak triggering cases of shearing tests, larger events are found more likely to occur when LURR is high than the smaller events, indicating that although no overall tidal triggering is observed, it is still possible to observe some correlation prior to strong earthquakes.

Within our parameter ranges, a seemingly intrinsic phase shift between the peak seismicity rate and the peak amplitude of the perturbation stress, decided by $A /(k T)$, is observed in this simple elastic-brittle system. This phenomenon is also supported by experimental results (LOCKNER and BEELER 1999) and simulation (TSURUOKA et al., 2002). The height of the probability density function of seismicity, or degree of tidal triggering, is controlled by another parameter, $A T / k$. The physical mechanisms of these two parameters remain unclear, they deserve further study, both observationally and numerically.

The results in this paper present another possible reason to explain why tidal triggering has not been widely observed. According to Vidale (VIDALE et al., 1998), 
the preseismic stress rate is much higher (1,000 times) than the long-term tectonic rate, subsequently the effect of tides is lessened. Our simulations do not contradict this idea, for the high preseismic stress rate means low $k_{3}$ in our study, therefore low correlation. A mechanism for delayed failure has been suggested to explain the observations (LOCKNER and BEELER 1999) and such a mechanism should be incorporated into the simulation model in future work. However, our studies suggest that even in elastic-brittle system without time-delayed failure, smaller period of perturbation $T$ can also contribute to weak correlation between tidal stress and seismicity. This also implied that when testing tidal triggering of earthquakes, it is necessary to devote attention to the longer period components of tidal stress instead of only the shortest one.

\section{Acknowledgments}

This study is supported by the Australia-China special Fund for Scientific and Technological Cooperation, UQ New Staff Research Start-up Fund Chinese NSF Fund for International Exchange and Cooperation and Chinese NSF (grant number 40004002). The authors thank two anonymous reviewers for their valuable advice enhancing this paper.

\section{REFERENCES}

BAI, Y. L. Wei, Y. J., XIA, M. F., and KE, F. J. (2000), Weibull Modulus for Diverse Strength due to Sample-specificity, Theoret. Appl. Fracture Mech. 34 (3), 211-216.

Bowman, D. D., Oulllon, G., Sammis, C. G., Sornette, A., and Sornette, D. (1998), An Observational Test of the Critical Earthquake Concept, J. Geophys. Res. 103, 24359-24372

Catton, L. A. (1922), Earthquake Frequency, with Special Reference to Tidal Stress in the Lithosphere, Bull. Seismol. Soc. Am. 12, 47-198.

Emter, D. (1997), Tidal Triggering of Earthquakes and Volcanic events, in (Wilhelm, H., et al. ed,) Tidal Phenomena, Springer-Verlag (1997), pp 293-309.

Heaton, T. H. (1982), Tidal Triggering of Earthquakes, Bull. Seismol. Soc. Am. 72, 2181-2200.

Locker, D. A. and Beeler, N. M. (1999), Premonitory Slip and Tidal Triggering of Earthquakes, J. Geophys. Res. 104, 20,133-20,151.

Mora, P. and Place, D. (2002a), Stress Correlation Function Evolution in Lattice Solid Elasto-dynamic Models of Shear and Fracture Zones and Earthquake Prediction, Pure Appl. Geophys. 159, 2413-2427.

Mora, P. Wang, Y. C Yin, C. Place, D., and Yin, X. C. (2002b), Simulation of the Load-unload Response Ratio and critical Sensitivity in the Lattice Solid model, Pure Appl Geophys. 159, 2525-2536.

Place, D. and Mora, P., (2001), A random lattice solid model for simulation of fault zone dynamics and fracture processes. In Bifurcation and Localisation Theory for Soils and Rocks'99, (eds. Mühlhaus, H-B., Dyskin, A. V. and PASternak, E.) (AA Balkema, Rotterdam/Brookfield 2001).

Place, D., Lombard, F., Mora, P., and Abe, S., 2002, Simulation of the Micro-physics of Rocks Using LSmearth, Pure Appl Geophys., 159, 1933-1950.

Rydelek, P. A., SaCKS, I. S., and ScARPa, R. (1992), On Tidal Triggering of Earthquake Swarms at Campi Flegrei, Geophys. J. Int. 109, 125-137. 
Tolstoy, M., Vernon, F. L., Orcutt, J. A. and Wyatt, F. K., (2002), Breathing of the Seafloor: Tidal Correlations of Seismicity at Axial Volcano, Geology 30, 503-506.

Tsuruoka, H. and Ohtake, M., 2002, Effects of the Earth Tide on Earthquake Occurrence: An Approach by Numerical Simulation, J. of Geography 111, 256-267. (Japanese with English abstract).

Tsuruoka, H., Ohtake, M., Sato, H. (1995), Statistical Test of the Tidal Triggering of Earthquakes: Contribution of the Ocean Tide Loading Effect, Geophys. J. Int. 122, 183-194.

Vidale, J. E., Agnew, D. C., Johnston, M. J. S., and Oppenheimer, D.H.1998, Absence of Earthquakes Correlation with Earth Tides: An Indication of High Preseismic Fault Stress rate, J. Geophys. Res. 103, 24,567-24,572.

William, S. and Wilcock, D, 2001, Tidal Triggering of Microearthquakes on the Juan de Fuca Ridge, Geophys. Res. Lett. 28, 3999-4002.

XIA, M. F., Song, Z. Q., Xu, J. B., ZhaO, K. H., and BAI, Y.L. (1996), Sample-specific Behavior in Failure Models of Disordered Media, Communications in Theoretical Physics, 25(1), 49-54.

Yin, X. C., Chen, X. Z., Song, Z. P., and Yin, C. (1995), A New Approach to Earthquake Prediction: the Load Unload Response Ratio (LURR) Theory, Pure Appl. Geophys. 145, 701-715.

Yin, X. C., Wang, Y. C., Peng, K. Y., BAi, Y. L. (2000), Development of a New Approach to Earthquake Prediction: Load/Unload Response Ratio (LURR) Theory, Pure Appl. Geophys. 157, 2365-2383.

Yin, X. C., Mora, P., Peng, K. Y., Wang, Y. C., and Weatherley, D. (2002), Load-unload Response Ratio and Accelerating Moment/Energy Release critical Region Scaling and Earthquake Prediction, Pure Appl. Geophys. 159, 2511-2523.

(Received September 27, 2002, revised January 27, 2003, accepted February 10, 2003)

To access this journal online:
http://www.birkhauser.ch

